\title{
I feel who I see: Visual body identity affects visual-tactile integration in peripersonal space
}

\author{
R. Salomon ${ }^{\mathrm{a}, *}$, M. van Elk ${ }^{\mathrm{a}}$, J.E. Aspell ${ }^{\mathrm{b}}$, O. Blanke ${ }^{\mathrm{a}, \mathrm{c}, \mathrm{d}}$ \\ ${ }^{a}$ Laboratory of Cognitive Neuroscience, Ecole Polytechnique Fédérale de Lausanne (EPFL), Lausanne 1015, Switzerland \\ ${ }^{\mathrm{b}}$ Department of Psychology, Anglia Ruskin University, Cambridge CB1 1PT, UK \\ ${ }^{\mathrm{c}}$ Department of Neurology, University Hospital, Geneva 1211, Switzerland \\ ${ }^{\mathrm{d}}$ Center for Neuroprosthetics, School of Life Sciences, Ecole Polytechnique Fédérale de Lausanne, Switzerland
}

\section{A R T I C L E I N F O}

\section{Article history:}

Received 13 January 2012

Available online 23 July 2012

\section{Keywords:}

Crossmodal integration

Visual tactile integration

Self-consciousness

Bodily representation

Social cognition

Self-representation

Crossmodal congruency

\begin{abstract}
A B S T R A C T
Recent studies have shown the importance of integrating multisensory information in the body representation for constituting self-consciousness. However, one idea that has received only scant attention is that our body representation is also constituted by knowledge of bodily visual characteristics (i.e. 'what I look like'). Here in two experiments we used a full body crossmodal congruency task in which visual distractors were presented on a photograph of the participant, another person, who was either familiar or unfamiliar, or an object. Results revealed that during the 'self-condition' CCEs were enhanced compared to the 'other condition'. The CCE was similar for unfamiliar and familiar others. CCEs for the object condition were significantly smaller. The results show that presentation of an irrelevant image of a body affects multimodal processing and that the effect is enhanced when that image is of the self. The results hold intriguing implications for body representation in social situations.
\end{abstract}

(c) 2012 Elsevier Inc. All rights reserved.

\section{Introduction}

How the brain represents and integrates bodily information from different sensory modalities while taking into account previous knowledge to give rise to our bodily consciousness is not well understood. The integration of bodily signals such as touch and proprioception with external information from vision has been studied extensively during the past few years (Holmes, Sanabria, Calvert, \& Spence, 2007; Macaluso, Frith, \& Driver, 2002; Shore, Barnes, \& Spence, 2006; Spence et al., 2004). These investigations were spurred by studies using single cell recordings in animals which revealed neurons that have receptive fields that respond to both visual and tactile stimuli (Graziano, Yap, \& Gross, 1994; Mountcastle, Lynch, Georgopoulos, Sakata, \& Acuna, 1975) as well as data from right brain-damaged patients with abnormalities in visuo-tactile integration (di Pellegrino, Làdavas, \& Farné, 1997; Làdavas, Pellegrino, Farnè, \& Zeloni, 1998). Subsequent studies suggested that similar multimodal representations of personal and peripersonal space can also be studied in healthy participants (Lloyd, Shore, Spence, \& Calvert, 2002; Macaluso, Frith, \& Driver, 2000; Spence, Pavani, Maravita, \& Holmes, 2004). One well established paradigm to quantitatively test such multimodal representations in humans is the crossmodal congruency task (Maravita, Spence, \& Driver, 2003; Spence, Pavani, \& Driver, 1998; Spence, Pavani, Maravita, \& Holmes, 2004). In the crossmodal congruency task participants are required to report the location of a tactile stimulation (up or down) while an irrelevant visual distractor is flashed at either the same spatial elevation (congruent condition) or the different elevation

\footnotetext{
* Corresponding author. Address: Laboratory of Cognitive Neuroscience, Ecole Polytechnique Fédérale de Lausanne (EPFL), SV BMI LNCO AAB 201 (Batiment AAB), Station 15, CH-1015 Lausanne, Switzerland. Fax: +41 216931770.

E-mail address: Roy.salomon@epfl.ch (R. Salomon).
} 
(incongruent condition). The basic finding - termed the crossmodal congruency effect (CCE) - is of slower reaction times (RTs) and/or more errors when the visual and tactile stimuli are incongruent. Furthermore, a larger CCE is observed when the visual distractor occurs on the same spatial side as the vibrotactile stimulation (e.g. distractor at lower left side, vibration at upper left side) (Spence, Pavani, \& Driver, 2004).

The CCE can be used as a measure of the crossmodal mapping of peripersonal space and to study the extension of personal space such as during the incorporation of external objects (tools or fake hands) into the body representation (Spence, Pavani, Maravita, et al., 2004). For example, a CCE (that is generally found between vibrations and light in close proximity to the participant's hands) has been shown when the visual distractors were located on rubber hands, but only when these were placed in a plausible position in relation to the participant's own hands (Pavani, Spence, \& Driver, 2000; Zopf, Savage, \& Williams, 2010). Similarly, CCEs have been found for distractors placed at the end of elongated tools, but only after participants had practiced using the tool (Holmes, Calvert, \& Spence, 2007; Maravita, Spence, Kennett, \& Driver, 2002). This finding has been interpreted as reflecting an extension of peripersonal space to incorporate the tool, comparable to the finding of an increase in the receptive field size of visual-tactile neurons following tool use in monkeys (but see Holmes, Sanabria, et al. 2007; Iriki, Tanaka, \& Iwamura, 1996). In addition, these CCE effects are not limited to the hands and handheld tools. For example, CCEs were also modulated by visual distractors displayed on the trunk of a body that was viewed via a video camera from a distance of $2 \mathrm{~m}$ and from behind. These CCEs were further modulated by visuo-tactile stroking, decreased for non-bodily control objects and enhanced when viewing and self-identifying with the seen human body (Aspell, Lenggenhager, \& Blanke, 2009; Palluel, Aspell, \& Blanke, 2011). Thus, events occurring in our extra-personal and peripersonal space may affect the way in which we experience our body. The body representation seems to be highly malleable (Holmes \& Spence, 2004), and under certain conditions it can be extended to include objects (Armel \& Ramachandran, 2003; Maravita et al., 2002), body parts (Botvinick \& Cohen, 1998) or even full bodies (Aspell et al., 2009; Lenggenhager, Mouthon, \& Blanke, 2009). Yet despite this flexibility, under normal conditions we rarely confuse our bodies with other objects or the bodies of others suggesting that the malleability of the body representation is constrained by additional mechanisms.

An important aspect of maintaining a coherent body representation is our ability to distinguish between what belongs to our own body proper and what does not (i.e. we take off our hat before combing our hair and we typically do not hesitate to poke in a fire with a stick, although we would not do such a thing with our finger) (Boinski, 1988). Several studies have shown that our proficiency in distinguishing ourselves from others relies on the use of visual information, by means of a comparison between online visual information and pre-existing knowledge of our body's visual appearance, which we will refer to here as 'visual body identity'. This ‘visual body identity' includes the stored knowledge of our external appearance that allows one to identify oneself in a photograph. The visual body identity can be considered a perceptual element of the more general concept of the 'body image' which has been defined as the perceptual, conceptual and emotional representations of the body which are not related to action (de Vignemont, 2010). Many studies have focused on the behavioral and neural mechanisms underlying the visual recognition of one's own face (Devue \& Bredart, 2011; Dieguez, Scherer, \& Blanke, 2011; Kircher et al., 2000; Platek et al., 2006; Uddin, Kaplan, Molnar-Szakacs, Zaidel, \& Iacoboni, 2005) and body (Frassinetti, Ferri, Maini, Benassi, \& Gallese, 2011; Frassinetti et al., 2009; Myers \& Sowden, 2008; Sugiura et al., 2006). For example, one study investigated the neural correlates of recognition of the self, a familiar other and a stranger from facial and body movies and pictures. The results indicated that self-recognition was faster than recognition of a stranger, for both faces and bodies, regardless of stimulus type (Sugiura et al., 2006). Several other studies have shown an advantage for processing and recognition of the own body (Devue et al., 2007) and body parts (Frassinetti et al., 2009; Salomon, Malach, \& Lamy, 2009). Taken together these findings show that our own visual body image (face, body or body parts) enjoys privileged processing which is governed by specific brain mechanisms. This self-representation may serve to constrain changes in body representation allowing us to differentiate our body from the environment.

Thus, on the one hand, studies of multisensory integration provide evidence for the flexibility of our body representation, while on the other hand, other studies show a privileged processing of visual information related to one's own body, suggesting the involvement of long-term visual knowledge in the body representation. Relatively little is known about how such body knowledge interacts with the integration of multisensory information related to one's body. For instance, when we are combing our hair in front of a mirror we need to integrate visual information about our body with multisensory information about the relative positions of our body parts. Such a seemingly simple task could easily go wrong, for instance when we are standing in front of a laughing mirror, causing us to be mistaken about the actual position of our body parts and thereby underlining the importance of long-term visual knowledge for a coherent body representation.

In the present study, we tested if crossmodal integration (the CCE) was modulated when an image of a body was viewed and whether this was further modulated by the identity of the body (i.e. whether the body belongs to me or not). Participants performed a full body crossmodal congruency task in which the visual distractors were superimposed on a picture of themselves or another person displayed on a large computer screen. We tested if images of the own body induced larger CCEs than images of another person or of an object. In the first experimental condition participants observed their own picture or that of an unfamiliar other. In the second experimental condition a second group of participants observed their own picture or that of a familiar person. In this way we controlled for the possible confound that eventual differences in the CCE are partly driven by familiarity (i.e. own body image is more familiar than the image of a stranger's body) (Dieguez et al., 2011) rather than by the identity of the body alone. In an additional experiment we tested whether CCEs would be larger for bodies then for a body sized object. Based on previous studies showing that the degree of identification with an external 
object (hand or full body) modulates CCE magnitude and hence its "incorporation" into a person's body representation (Aspell et al., 2009; Zopf et al., 2010) (despite controlling for low-level factors such as size, clothing and posture), we expected higher CCEs in the self-condition compared to the other condition and higher CCE's when viewing people than when viewing an object.

\section{Methods}

\subsection{Participants}

Thirty healthy volunteers ( 10 females, mean age 22.8 years, s.d. 3 years) participated in the first experiment, and another 17 participants ( 8 females, mean age 22.8 years, $S D=3$ years) participated in the second experiment. The studies were approved by the local ethics committee: La Commission d'ethique de la recherche Clinique de la Faculte de Biologie et de Medecine - at the University of Lausanne, Switzerland. All participants gave informed consent and were fully debriefed. After the experiment the participants filled out a short questionnaire to assess their identification with the pictures shown and to collect some demographic information. All participants had no previous experience with the task, had normal or corrected to normal vision and had no history of neurological or psychiatric conditions.

\subsection{Experimental procedure and apparatus}

\subsubsection{Experiment 1}

Participants first changed into a white t-shirt and a full body photograph on a fixed background was taken from a distance of $2 \mathrm{~m}$ (Canon PowershotSX200). These photographs were then resized (Adobe Photoshop CS5) to fit a standard body template so that all bodies were the same size on the screen. For each participant the picture was mirror-reversed, so that the picture on the screen would be to the same as the participant's mirror image. The participants were then fitted with four tactile vibrators each consisting of a small vibrating motor (Precision MicroDrives shaftless vibration motors, model $312-101,3 \mathrm{~V}, 60 \mathrm{~mA}, 9000 \mathrm{rpm}(150 \mathrm{~Hz}), 5 \mathrm{~g})$. The motors had a surface area (the area touching the skin) of $113 \mathrm{~mm} 2$. These were placed on predefined anatomical locations: left and right shoulder $(2 \mathrm{~cm}$ under the clavicle), left and right pelvis ( $4 \mathrm{~cm}$ above the pelvic bone). The participants then placed their right foot on two response pedals (Psychology Software Tools, Inc., Pittsburgh, PA), facing a 50 inch inverted television screen (Samsung N002 HD) placed on the wall $2 \mathrm{~m}$ away (see Fig. 1). The experiment was run using Presentation software (Neurobehavioural systems, Albancy, CA). During the experiment participants viewed a picture of themselves or a picture of a gender- matched partner, which was changed every 5 trials. To avoid habituation effects for identity, the two experimental conditions regarding the familiarity of the other (i.e. familiar and unfamiliar other) were run using separate groups of participants in a mixed within and between subject design. In the first experimental condition participants observed pictures of themselves and of another participant that they did not know. In the second experimental condition participants were required to come to the lab together with a friend they had known for at least 5 months. Accordingly, in the second experimental condition participants observed pictures of themselves and of their friend. The rationale for manipulating the familiarity of the other person in the picture was to investigate if eventual differences between the CCE in response to self and other pictures might be mediated by the familiarity of the picture (i.e. you have more experience with observing your own body than a stranger's body). In order to avoid strong block-order effects we decided to alternate self and other pictures in mini-blocks of 5 trials.

A central fixation cross was placed in the center of the picture. Four white asterisks serving as visual cues (60 $\times 60$ pixels) were presented at locations corresponding to the locations of the tactile stimulators (left and right shoulder, left and right pelvis). On each trial one of the asterisks changed color to red for three epochs of $50 \mathrm{~ms}$ interleaved with three tactile stimulations of $50 \mathrm{~ms}$ each (Fig. 1). The locations of the visual and tactile stimulations as well as block order were randomized. The experiment consisted of 384 trials with 96 repetitions per condition (self/other $\times$ congruent/incongruent) The participants were required to indicate by lifting the foot from the response pedal whether the tactile stimulation was at one of the top locations (shoulders) or bottom locations (pelvis). Participants were requested to respond as accurately and quickly as possible.

\subsubsection{Experiment 2}

In the second experiment, participants saw the visual distractors on a picture of their own body, an unfamiliar other or a body sized object. The object was a grey rectangle (RGB:187,179,179) with the same size as the body template used in the other conditions. As in the first experiment, a central fixation cross was placed in the horizontal center of the picture. Four white asterisks serving as visual cues $(60 \times 60$ pixels) were presented at locations corresponding to the locations of the tactile stimulators. The experiment included 98 trials per condition (self/other/object $\times$ congruent/incongruent) with 588 trials overall. All other parameters were identical to experiment 1 . Following the experiment participants were debriefed and asked to fill out a questionnaire relating to the visual similarity between themselves and the other participant. A subset of participants from the first experiment was also requested to fill in the questionnaire. 
a

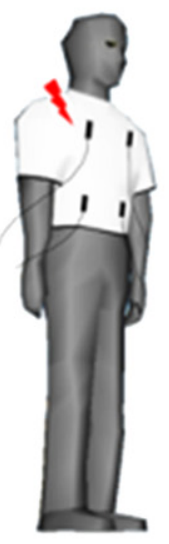

b

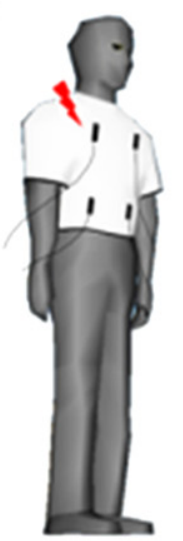

C

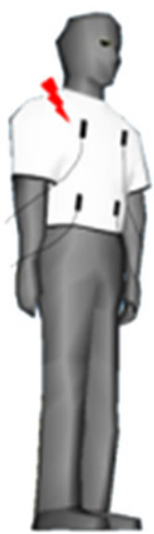

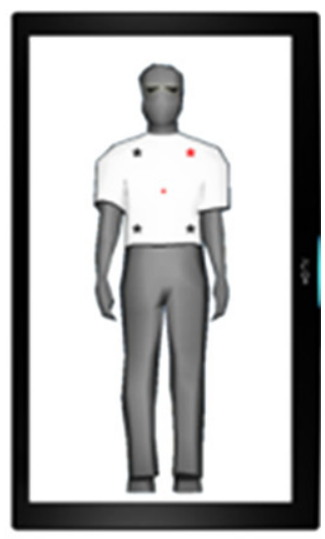
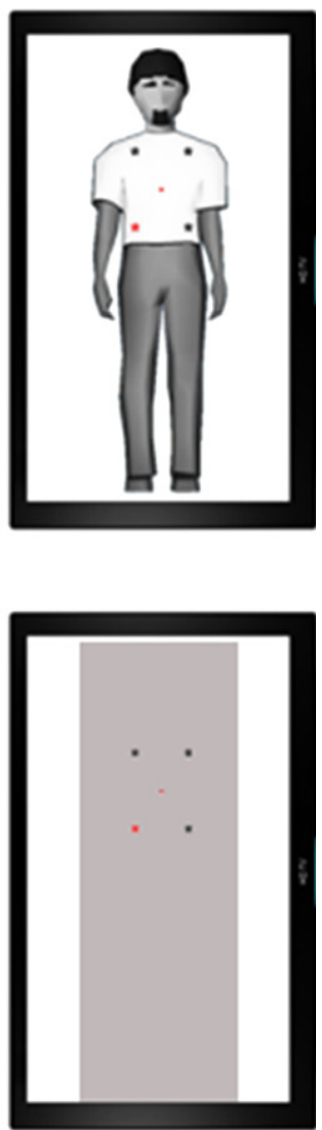

Fig. 1. Experimental paradigm. Participants stood facing an inverted 50 in. television screen. Tactile stimulators were placed in four standard locations on the front of the participants' body. On half of the trials the background picture was a picture of the participant (self condition-panel a) and in the other half of the trials the picture was of another person (other condition-panel b). On each trial an irrelevant visual distractor light flashed (red asterisk) in one of four possible locations corresponding to the locations of the tactile stimulators on the participants' body while simultaneously a tactile stimulation was delivered to one of the vibrators (shown for illustration purposes as red bolts). Participants had to respond as quickly as possible to the location (up or down) of the tactile vibration while ignoring the visual distractor. Congruent trials (top panel) are those in which the visual and tactile stimulation were at same elevation (top/bottom). Example of an incongruent trial is shown in panel b. An additional object condition was included in experiment 2 (panel c). (For interpretation of the references to colour in this figure legend, the reader is referred to the web version of this article.) 


\section{Results}

\subsection{Experiment 1}

Trials in which the participants failed to respond within $1500 \mathrm{~ms}$ (less than $1 \%$ of trials) and trials with incorrect responses or trial were discarded from the reaction time analysis. RT outliers (more than 2 STDs from the participant's mean RT) were also discarded (less than $5 \%$ of trials). Mean accuracy and RTs for all categories are summarized in Table 1 . The accuracy and reaction time (RT) data were combined to a single measure of 'Inverse Efficiency' (IE) by dividing the reaction times by the proportion of correct responses for each participant in each condition. This dependent measure allows us to correct for any instances of speed-accuracy trade-offs (Ehrsson, Spence, \& Passingham, 2004; Shore et al., 2006; Spence, Kingstone, Shore, \& Gazzaniga, 2001; Spence, Pavani, Maravita, et al., 2004). The IE data was analyzed using a mixed 4 way ANOVA with 3 within participant factors: identity (self/other), side (same/different) and congruency (congruent/incongruent). A between participant factor of manipulation examined the effect of the other's identity (unfamiliar/familiar) which was the single difference between the two experimental conditions.

In line with our prediction a significant interaction between identity and congruency was revealed $(F(1,29)=4.6, p<.05)$. Planned comparisons revealed that the CCE was larger when participants viewed their own picture $(M=216)$ then when they viewed another person's picture $(M=179)(t(1,29)=2.2, p<.05)$ (see Fig. 2$)$. To further explore the origin of this interaction we investigated the mean IEs for self and other by congruency (Fig. 3). This revealed that the interaction was driven by a large difference in the incongruent condition (self-incongruent - other incongruent $=48 \mathrm{~ms}$ ) while the difference in the congruent condition was much smaller (self-congruent - other congruent $=10 \mathrm{~ms}$ ). Interestingly, this effect was not modified by the familiarity of the other's identity as no three way interaction was found between identity, congruency and experimental condition $(F(1,29)=0.4$, n.s. $)$.

In addition, the results showed a main effect for identity, $(F(1,29)=18.8, p<.000)$, with participants being faster to respond to non-self stimuli than to their own picture $\left(M_{\text {non-self }}=945 ; M_{\text {self }}=974\right)$. As expected a main effect of congruency was found $(F(1,29)=54.9 ; p<.000)$, with participants being faster to respond in the congruent condition $(M=861)$ than the incongruent condition $(M=1058)$. A main effect for side was also found with participants responding slower to stimuli on the same side as the visual distractor $(F(1,29)=6.70, p<.05)\left(M_{\text {same }}=973 ; M_{\text {different; }}=946\right)$. As expected an interaction between congruency and side was also found, $(F(1,29)=4.99, p<.05)$, with larger CCEs on the same side $(M=215)$ than on different sides $(M=180)$.

Finally, we tested if the CCE for the non-self-picture (familiar or unfamiliar) was significant. The "other" CCE IE ('other incongruent' minus 'other congruent') for all participants was used in a single sample $t$-test. The results clearly showed that the CCE in the other condition was significant $(t(29)=6.3, p<.000(M=178))$.

\subsection{Experiment 2}

Two participants were removed from the analysis due to a technical issue during the experiment (a problem with one tactile vibrator). Data from another participant was not included as he indicated that he was familiar with the unfamiliar other. Thus in total, data from 14 subjects was analyzed in the second experiment.

The results of experiment 2 were preprocessed as in experiment 1. RT outliers comprised less than 3\% of the data. Mean accuracy for the second experiment was $94.2 \%$. The results were analyzed using 3-way repeated measures ANOVA with the factors: identity (self/other/object), side (same/different) and congruency (congruent/incongruent) on the IE data.

The results revealed the critical interaction between identity and congruency $(F(2,26)=11.3, p<.001)$. Planned comparisons between CCEs for the identity conditions showed that this was driven by a difference between self $(M=153)$ and object

Table 1

Reaction time, accuracy and inverse efficiency by experiment and condition.

\begin{tabular}{|c|c|c|c|c|c|c|c|c|}
\hline & Cong & Incong & Same side & Diff. side & Self & Other (Stranger) & Self & Other (Familiar) \\
\hline \multicolumn{9}{|c|}{ Experiment 1} \\
\hline RT (ms) & 794 & 877 & 840 & 832 & 833 & 828 & 846 & 838 \\
\hline STD & 136 & 160 & 157 & 151 & 146 & 142 & 164 & 164 \\
\hline ACC $\%$ & 92.5 & 84.3 & 87.9 & 88.9 & 89.6 & 90.4 & 85.6 & 88 \\
\hline STD & 4.1 & 9.1 & 8.6 & 7.6 & 6.6 & 5.4 & 10.1 & 9 \\
\hline IE (ms) & 861 & 1058 & 973 & 946 & 940 & 922 & 1009 & 967 \\
\hline \multirow[t]{2}{*}{ STD } & 155 & 255 & 254 & 209 & 209 & 189 & 267 & 253 \\
\hline & Cong & Incong & Same Side & Diff. Side & Self & Other (Stranger) & Object & \\
\hline \multicolumn{9}{|c|}{ Experiment 2} \\
\hline RT (ms) & 723 & 808 & 766 & 764 & 720 & 770 & 806 & \\
\hline STD & 109 & 142 & 136 & 131 & 109 & 134 & 142 & \\
\hline ACC $\%$ & 97.1 & 92.9 & 94.2 & 95.8 & 96.9 & 94.9 & 93.1 & \\
\hline STD & 2.8 & 5.4 & 4.5 & 4.9 & 3 & 5.1 & 5.1 & \\
\hline $\mathrm{IE}(\mathrm{ms})$ & 745 & 873 & 817 & 801 & 743 & 815 & 869 & \\
\hline STD & 116 & 166 & 163 & 151 & 117 & 158 & 167 & \\
\hline
\end{tabular}




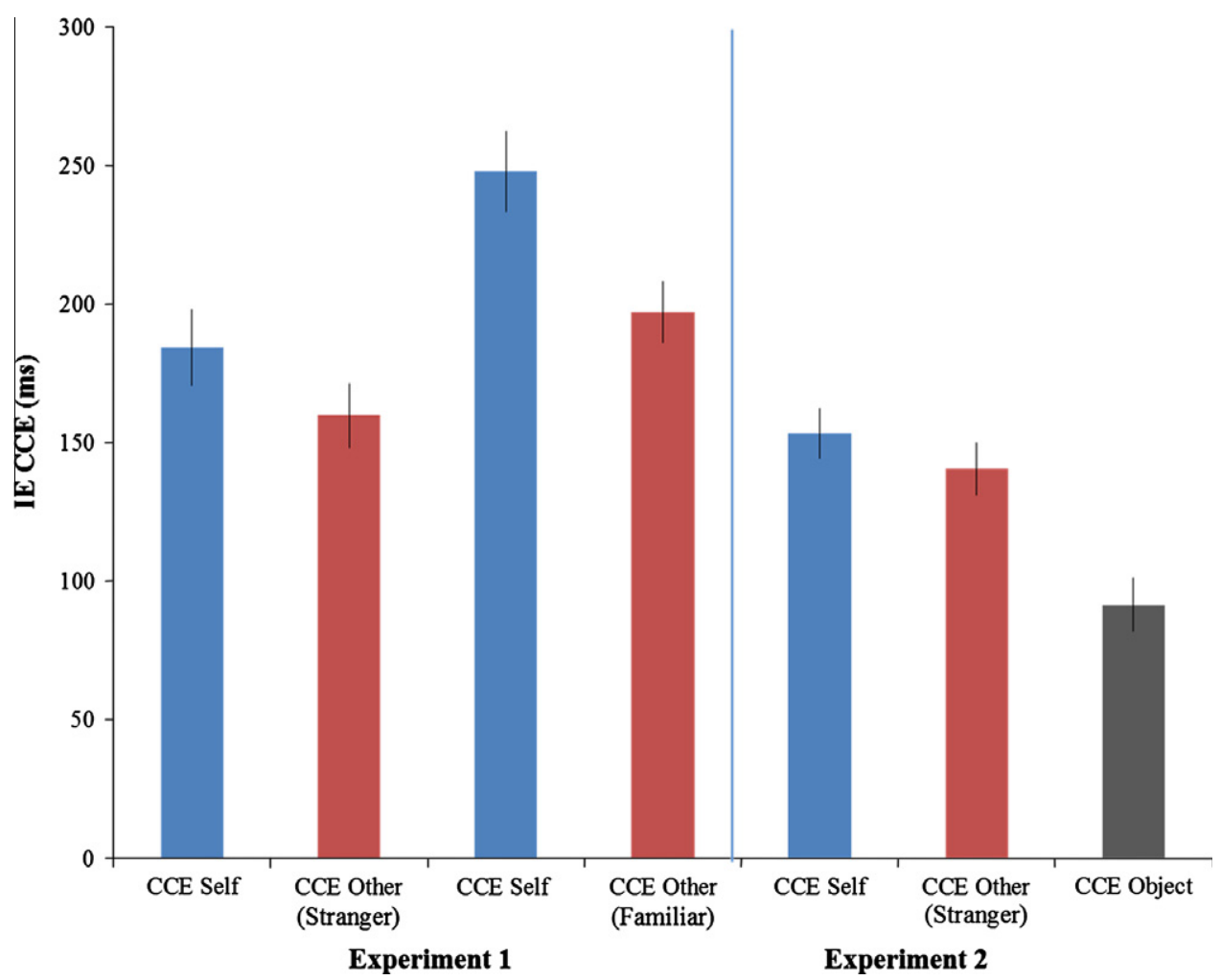

Fig. 2. Crossmodal congruency effect by identity. Inverse efficiency CCE (incongruent-congruent) by identity of background picture: (self/other (unfamiliar) and (self/other (familiar) experiment 1 and (self/other (unfamiliar)/object) experiment 2. Error bars represents confidence intervals (Loftus \& Masson, 1994).

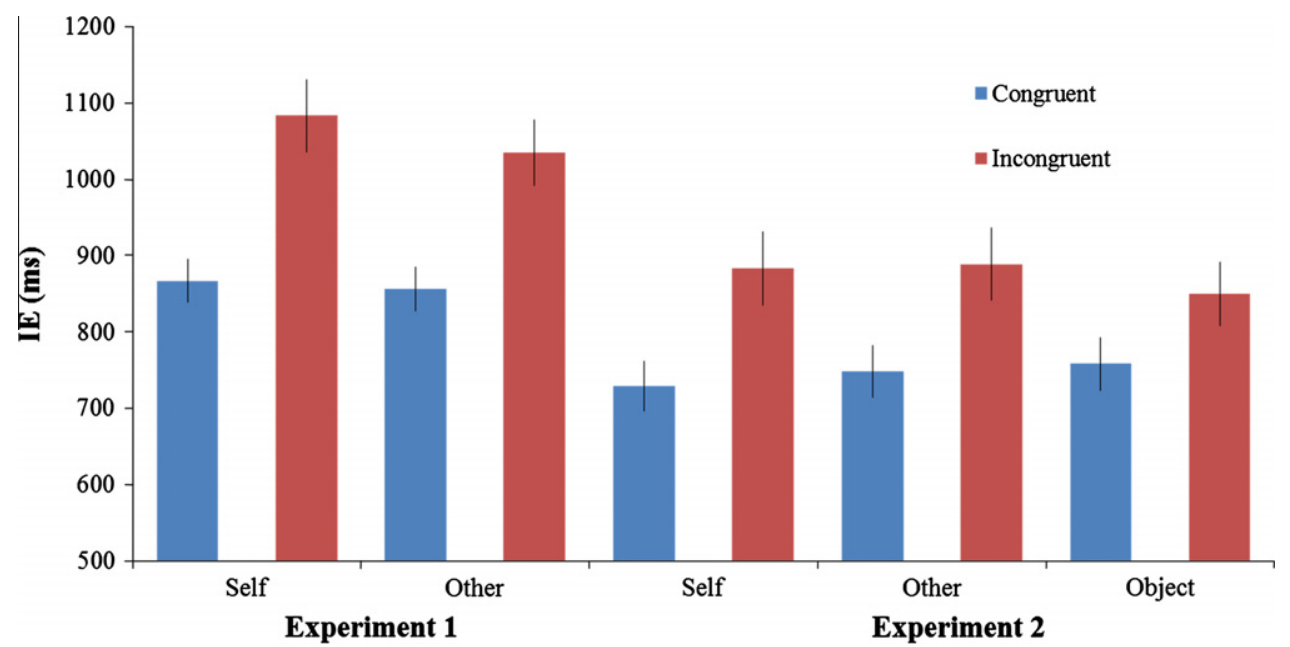

Fig. 3. Inverse efficiency by identity and congruency. Mean inverse efficiency by identity (self/other) and congruency (congruent/incongruent) in experiment 1 and (self/other/object) experiment 2. Note similar IEs were found in congruent conditions for both identities in experiment 1 . Error bars represents confidence intervals (Loftus \& Masson, 1994).

$(M=91)(t=3.6, p<.01)$ and between other $(M=141)$ and object $(t=3.4 p<.01$, Bonferroni corrected $)$. A trend for differences between self and other was also found $(t=2.9 p=.08$ ), with self CCEs being larger than CCEs for others (Fig. 2). A main effect for congruency was also found $(F(1,13)=45.5 p<.0001)$ with congruent responses $(M=745)$ being shorter than incongruent trials $(M=874)$. The effect of side was also significant $(F(1,13)=6.8, p<.05)$ with same side responses being smaller $(M=817)$ than different side responses $(M=802)$. No other effects reached significance (all $p>.19)$. Additionally, we tested if 


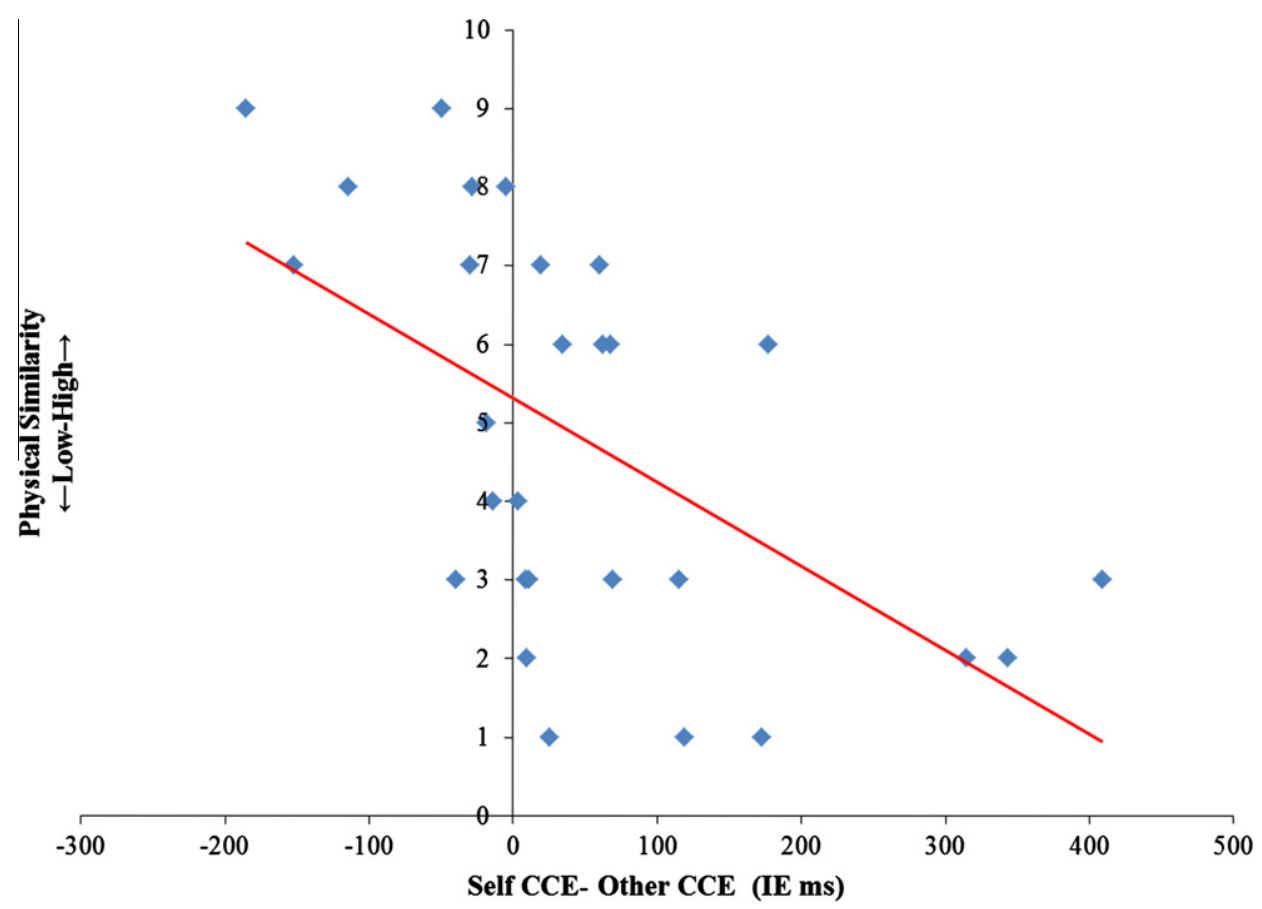

Fig. 4. Correlation between physical similarity and self CCE bias. The figure depicts the correlation between subject post hoc self-ratings of physical similarity with the picture shown in the other condition and the self CCE bias (self CCE-other CCE). Note as the rating of physical similarity increased smaller differences were found between the self and other conditions $(r=-.56, p=.0008)$. The red line plots the linear trend of the data. (For interpretation of the references to colour in this figure legend, the reader is referred to the web version of this article.)

the CCE for the object condition was significant. The object CCE IE for all participants was used in a single sample $t$-test. The results indicated that the CCE in the object condition was significant $(t(13)=6.5, p<.000(M=91 \mathrm{SD}=51.9))$.

Finally, we requested a subset of the participants from both experiments $(N=28)$ to rate the level of physical similarity between themselves and the "other" on a 10 -point scale $(1=$ not similar, $10=$ very similar $)$. We performed correlation analysis between these ratings and the magnitude of the difference between the self CCE and other CCE for each subject. The result showed a strong negative correlation $(r=-.56 p=.0008)$ indicating that the level of perceived physical similarity between the self and other was related to the amount of interference from the identity of the picture (Fig. 4). That is, participants who perceived themself to be more similar to the other had a CCE for the other that was comparable in size to the self CCE and vice versa. To further test this correlation we divided the subjects in two groups based upon a median split of the ratings of perceived physical similarity, thus creating a low similarity group (rating $1-5, N=13$ ) and a high similarity group (rating 5-10,N=15). The mean difference between the self CCE and other CCE for the low similarity group was considerably lower $(M=-10.9 \mathrm{SD}=95.3)$ than in the high similarity group $(M=102 \mathrm{SD}=139.8)$. A $t$-test between the scores of the two groups revealed a significant difference $(t=2.36, p<.05)$.

\section{Discussion}

In the present study, we investigated if and to what extent crossmodal integration is modulated by the identity of a visually presented body. At least four main findings support the notion that visuo-tactile spatial integration is affected by the presence of the image of a person. First, CCEs were significantly higher when the visual distractors were presented on an image of a body rather than a body sized object. Second, the identity of the seen body also affected visual tactile integration: visual distractors placed on a self-picture caused more crossmodal interference than distractors placed on a picture of another person, independent of whether the stimuli were applied on the same or on different sides. Third, the perceived level of physical similarity between the participant and that of the other as judged by the participants correlated with their CCE scores. Participants who rated themselves as dissimilar to the other person showed smaller CCEs in the other condition compared to the self-condition (Fig. 4). In contrast, participants who rated themselves as similar to the other person showed CCEs that were comparable in size for self and other pictures. Fourth, the results show that the enhanced crossmodal integration for self-pictures is not due to the familiarity with the depicted body, as the CCE did not differ when the other's picture was of a highly familiar person. Together these findings show that the identity of a full body representation affects crossmodal integration as measured by visuo-tactile CCE magnitude. 
Our results show that the CCE was stronger when the visual distractors were superimposed on bodily compared to nonbodily objects. This is in line with previous results using human full body crossmodal integration paradigms that showed no CCE or crossmodal priming effects when the distractor cues were projected on a non-body surface (Aspell et al., 2009; Thomas, Press, \& Haggard, 2006). However, the results indicated that the CCE for the object condition was still significant. This effect may be due to the specific experimental setup in which the background picture was changed every five trials (unlike the studies cited above which employed blocks of body vs. object trials). This rapid switching of the background picture may have caused some residual embodiment with the object due to the highly similar context. Results from several experiments have shown that the visual characteristics of the real and virtual bodies or body parts need not be identical in order to induce illusory body ownership (i.e. illusions have been induced with pictures of another person (Ionta et al., 2011) and a gender mismatched avatar (Slater, Spanlang, Sanchez-Vives, \& Blanke, 2010). Hence, a visual body representation may present a high level constraint required for induction of changes in bodily consciousness through multisensory integration (Tsakiris \& Haggard, 2005; but see Armel \& Ramachandran, 2003). Further experiments manipulating the levels of similarity between the observed and viewed bodies are required to elucidate the specific mechanisms by which visual body identity affects crossmodal integration.

In the present study, it was found that the CCE magnitude was modulated by the identity of the observed person. More specifically, a stronger CCE was observed when visual distractors were superimposed on a picture of oneself compared to another person. This finding indicates that the 'visual body identity' interacts with lower level multi-sensory processes related to the integration of the visual distractors with the tactile cues. As body size, posture and clothing were controlled it is likely that identity was most readily discerned from facial cues. Yet, why should the identity of the person depicted in the photo, used as the background for irrelevant visual distractors have such a strong effect on visual tactile integration? At least two related interpretations for these findings are possible: First, it may be that the visual self-image caused the participants to identify more with the external self-photo than with the other person's photo: this may in turn facilitate remapping of the visual and tactile stimulations and thus result in a stronger interference with the tactile discrimination. This explanation is in line with studies showing enhanced visuo-tactile interactions for felt tactile stimulations paired with the observation of touch on one's own compared to another person's face (Serino, Pizzoferrato, \& Ladavas, 2008). Alternatively, the increased physical compatibility in the self-condition may allow participants to extend or project their body representation to the visual image of themselves, thereby resulting in a stronger interference from the visual distractors. This interpretation is in line with previous findings showing that incorporation of other objects into the multimodal body representation is facilitated by the congruence between the object and body (Costantini \& Haggard, 2007).

We further found that the CCE difference between self and other pictures correlated strongly with the perceived level of similarity between the self and the other. That is, participants who considered themself to be physically similar to the other person showed a CCE to both pictures that was comparable in size, whereas participants who perceived themselves to be dissimilar showed a stronger CCE for self compared to other pictures. The finding that the CCE is modulated according to the physical similarity of a body picture to one's real body provides a further argument for the notion that our visual body-identity modulates crossmodal integration. We note that the participants in the present study came from a rather homogenous population, which may have reduced the visual differences between the self and other pictures. Ethnic selfother differences may further modulate crossmodal integration as shown previously (Serino, Giovagnoli, \& Làdavas, 2009).

In addition to these main findings, a few minor issues require discussion. First, the CCE was comparable in size when participants observed a body of a familiar and an unfamiliar person. This finding rules out the possible confound that the stronger CCE observed for pictures of oneself is related to the familiarity of one's self-representation (i.e. due to more experience with observing pictures of oneself or looking at oneself in the mirror). Another possible confound is that the self is a more interesting stimulus than the picture of another person causing participants to pay more attention to the picture during the self-condition. Yet, our analysis shows that generally the RTs of congruent trials in the self and other condition do not differ (Fig. 3) suggesting that attention to the self is not responsible for the larger crossmodal interference in the self-condition.

Overall participants responded slower to pictures representing their own body compared to the body of another person. This finding is in accord with previous studies showing a preferential processing of self-images compared to other images (Devue et al., 2007; Frassinetti et al., 2009, 2011; Knoblich \& Prinz, 2001; Serino et al., 2008; Sugiura et al., 2006; Uddin et al., 2005). Note that whereas most studies - in which the picture was relevant to the subject's task - have found a decrease in RT to self-images compared to images of others, the slower responses to self-images found in the present study were obtained in response to task-irrelevant stimuli. Hence enhanced processing of the self-image was associated with longer reaction times for the self-condition.

Studies on the full body illusion induced by synchronous visuo-tactile stimulation have also underlined the importance of identification with the visual body representation for visuo-tactile interactions (Ionta et al., 2011; Lenggenhager, Tadi, Metzinger, \& Blanke, 2007). In two studies which measured CCE during the full body illusion a larger CCE was found when participants self-identified with the viewed body as a result of congruent compared to incongruent visuo-tactile stimulation (Aspell et al., 2009; Palluel et al., 2011). Similar results have also been obtained in a study on the rubber hand illusion, where self-attribution of the fake rubber hand caused higher CCEs compared to a no illusion condition (Zopf et al., 2010). The present study shows that the mere presentation of a human body is already sufficient to facilitate crossmodal integration, even without inducing a full-body or rubber hand illusion. One possible explanation for this effect may be that because participants were presented with a front-view of the human with similar visual characteristics (i.e. posture, size, clothing), which 
may have resulted in a facilitated identification with the body in the picture - as if looking in a mirror or facing another person.

\section{Conclusions}

In conclusion, our data show that low-level processes affect the perception of our body but that this effect is modified by high-level representations such as our visual body identity. This interaction between low-level bottom-up multisensory processes and high-level top-down visual body representations (see also: Tsakiris \& Haggard, 2005) may allow us to retain an intact bodily representation in complex social situations, where we are exposed to conspecifics with highly similar bodies, yet distinct visual features. Thus, knowledge of our visual attributes is important to the shaping of our bodily experience in social situations.

\section{Acknowledgments}

We would like to thank Natalia Fernandez for helping with data acquisition. We are also grateful to the reviewers for their helpful comments.

\section{References}

Armel, K. C., \& Ramachandran, V. S. (2003). Projecting sensations to external objects: Evidence from skin conductance response. Proceedings of the Royal Society of London. Series B: Biological Sciences, 270(1523), 1499-1506.

Aspell, J., Lenggenhager, B., \& Blanke, O. (2009). Keeping in touch with one's self: Multisensory mechanisms of self-consciousness. PLoS ONE, 4(8), e6488.

Boinski, S. (1988). Use of a club by a wild white-faced capuchin (Cebus capucinus) to attack a venomous snake (Bothrops asper). American Journal of Primatology, 14(2), 177-179.

Botvinick, M., \& Cohen, J. (1998). Rubber hands' feel'touch that eyes see. Nature, 391(6669), 756.

Costantini, M., \& Haggard, P. (2007). The rubber hand illusion: Sensitivity and reference frame for body ownership. Consciousness and Cognition, 16(2), $229-240$.

de Vignemont, F. (2010). Body schema and body image-Pros and cons. Neuropsychologia, 48(3), 669-680.

Devue, C., \& Bredart, S. (2011). The neural correlates of visual self-recognition. Consciousness and Cognition, 20(1), 40-51.

Devue, C., Collette, F., Balteau, E., Degueldre, C., Luxen, A., Maquet, P., et al (2007). Here I am: The cortical correlates of visual self-recognition. Brain Research, $1143,169-182$.

di Pellegrino, G., Làdavas, E., \& Farné, A. (1997). Seeing where your hands are. Nature, 388(6644), 730.

Dieguez, S., Scherer, J., \& Blanke, O. (2011). My face through the looking-glass: The effect of mirror reversal on reflection size estimation. Consciousness and Cognition, 20(4), 1452-1459.

Ehrsson, H., Spence, C., \& Passingham, R. (2004). That's my hand! Activity in premotor cortex reflects feeling of ownership of a limb. Science, 305(5685), 875.

Frassinetti, F., Ferri, F., Maini, M., Benassi, M. G., \& Gallese, V. (2011). Bodily self: an implicit knowledge of what is explicitly unknown. Experimental Brain Research, 1-8.

Frassinetti, F., Pavani, F., Zamagni, E., Fusaroli, G., Vescovi, M., Benassi, M., et al (2009). Visual processing of moving and static self body-parts. Neuropsychologia, 47(8-9), 1988-1993.

Graziano, M., Yap, G. S., \& Gross, C. G. (1994). Coding of visual space by premotor neurons. Science, 266(5187), 1054.

Holmes, N. P., Calvert, G. A., \& Spence, C. (2007). Tool use changes multisensory interactions in seconds: evidence from the crossmodal congruency task. Experimental Brain Research, 183(4), 465-476.

Holmes, N. P., Sanabria, D., Calvert, G. A., \& Spence, C. (2007). Tool-use: Capturing multisensory spatial attention or extending multisensory peripersonal space? Cortex, 43(3), 469-489.

Holmes, N. P., \& Spence, C. (2004). The body schema and multisensory representation (s) of peripersonal space. Cognitive Processing, 5(2), 94-105.

Ionta, S., Heydrich, L., Lenggenhager, B., Mouthon, M., Fornari, E., Chapuis, D., et al (2011). Multisensory mechanisms in temporo-parietal cortex support selflocation and first-person perspective. Neuron, 70(2), 363-374.

Iriki, A., Tanaka, M., \& Iwamura, Y. (1996). Coding of modified body schema during tool use by macaque postcentral neurones. NeuroReport, 7(14), 2325.

Kircher, T., Senior, C., Phillips, M., Benson, P., Bullmore, E., Brammer, M., et al (2000). Towards a functional neuroanatomy of self processing: Effects of faces and words. Cognitive Brain Research, 10(1-2), 133-144.

Knoblich, G., \& Prinz, W. (2001). Recognition of self-generated actions from kinematic displays of drawing. Journal of Experimental Psychology Human Perception and Performance, 27(2), 456-465.

Làdavas, E., Pellegrino, G., Farnè, A., \& Zeloni, G. (1998). Neuropsychological evidence of an integrated visuotactile representation of peripersonal space in humans. Journal of Cognitive Neuroscience, 10(5), 581-589.

Lenggenhager, B., Mouthon, M., \& Blanke, O. (2009). Spatial aspects of bodily self-consciousness. Consciousness and Cognition, 18(1), 110-117.

Lenggenhager, B., Tadi, T., Metzinger, T., \& Blanke, O. (2007). Video ergo sum: Manipulating bodily self-consciousness. Science, $317(5841), 1096$.

Lloyd, D. M., Shore, D. I., Spence, C., \& Calvert, G. A. (2002). Multisensory representation of limb position in human premotor cortex. Nature Neuroscience, $6(1), 17-18$.

Loftus, G. R., \& Masson, M. E. J. (1994). Using confidence intervals in within-subject designs. Psychonomic Bulletin \& Review, 1(4), 476-490.

Macaluso, E., Frith, C. D., \& Driver, J. (2000). Modulation of human visual cortex by crossmodal spatial attention. Science, 289(5482), 1206.

Macaluso, E., Frith, C. D., \& Driver, J. (2002). Crossmodal spatial influences of touch on extrastriate visual areas take current gaze direction into account. Neuron, 34(4), 647-658.

Maravita, A., Spence, C., \& Driver, J. (2003). Multisensory integration and the body schema: Close to hand and within reach. Current Biology, 13(13), R531-R539.

Maravita, A., Spence, C., Kennett, S., \& Driver, J. (2002). Tool-use changes multimodal spatial interactions between vision and touch in normal humans. Cognition, 83(2), B25-B34.

Mountcastle, V. B., Lynch, J., Georgopoulos, A., Sakata, H., \& Acuna, C. (1975). Posterior parietal association cortex of the monkey: Command functions for operations within extrapersonal space. Journal of Neurophysiology, 38(4), 871.

Myers, A., \& Sowden, P. T. (2008). Your hand or mine? The extrastriate body area. Neuroimage, 42(4), 1669-1677.

Palluel, E., Aspell, J. E., \& Blanke, O. (2011). Leg muscle vibration modulates bodily self-consciousness: Integration of proprioceptive, visual, and tactile signals. Journal of Neurophysiology, 105(5), 2239.

Pavani, F., Spence, C., \& Driver, J. (2000). Visual capture of touch: Out-of-the-body experiences with rubber gloves. Psychological Science, 11(5), 353. 
Platek, S., Loughead, J., Gur, R., Busch, S., Ruparel, K., Phend, N., et al (2006). Neural substrates for functionally discriminating self-face from personally familiar faces. Human Brain Mapping, 27(2), 91-98.

Salomon, R., Malach, R., \& Lamy, D. (2009). Involvement of the intrinsic/default system in movement-related self recognition. PLoS ONE, 4(10), e7527.

Serino, A., Giovagnoli, G., \& Làdavas, E. (2009). I feel what you feel if you are similar to me. PLoS ONE, 4(3), e4930.

Serino, A., Pizzoferrato, F., \& Ladavas, E. (2008). Viewing a face (especially one's own face) being touched enhances tactile perception on the face. Psychological Science, 19(5), 434.

Shore, D. I., Barnes, M. E., \& Spence, C. (2006). Temporal aspects of the visuotactile congruency effect. Neuroscience Letters, 392(1-2), 96-100.

Slater, M., Spanlang, B., Sanchez-Vives, M. V., \& Blanke, O. (2010). First person experience of body transfer in virtual reality. PLoS ONE, 5(5), e10564.

Spence, C., Kingstone, A., Shore, D. I., \& Gazzaniga, M. S. (2001). Representation of visuotactile space in the split brain. Psychological Science, 12(1), 90.

Spence, C., Pavani, F., \& Driver, J. (1998). What crossing the hands can reveal about visuotactile links in spatial attention. Psychonomic Society, $3,13$.

Spence, C., Pavani, F., \& Driver, J. (2004). Spatial constraints on visual-tactile cross-modal distractor congruency effects. Cognitive, Affective, \& Behavioral Neuroscience, $4(2), 148$.

Spence, C., Pavani, F., Maravita, A., \& Holmes, N. (2004). Multisensory contributions to the 3-D representation of visuotactile peripersonal space in humans: Evidence from the crossmodal congruency task. Journal of Physiology-Paris, 98(1-3), 171-189.

Sugiura, M., Sassa, Y., Jeong, H., Miura, N., Akitsuki, Y., Horie, K., et al (2006). Multiple brain networks for visual self-recognition with different sensitivity for motion and body part. Neuroimage, 32(4), 1905-1917.

Thomas, R., Press, C., \& Haggard, P. (2006). Shared representations in body perception. Acta Psychologica, 121(3), 317-330.

Tsakiris, M., \& Haggard, P. (2005). The rubber hand illusion revisited: Visuotactile integration and self-attribution. Journal of Experimental Psychology: Human Perception and Performance, 31(1), 80.

Uddin, L., Kaplan, J., Molnar-Szakacs, I., Zaidel, E., \& Iacoboni, M. (2005). Self-face recognition activates a frontoparietal" mirror" network in the right hemisphere: an event-related fMRI study. Neuroimage, 25(3).

Zopf, R., Savage, G., \& Williams, M. A. (2010). Crossmodal congruency measures of lateral distance effects on the rubber hand illusion. Neuropsychologia, $48(3), 713-725$. 